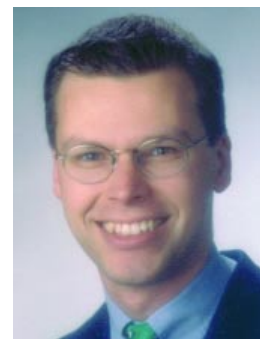

Oliver Neuhaus

\title{
Cannabinoide - symptomatische Therapie der Multiplen Sklerose?
}

\author{
Oliver Neuhaus und Hans-Peter Hartung \\ Neurologische Klinik, Heinrich-Heine-Universität, Düsseldorf
}

psychoneuro 2004; 30 (7): 389-392

$\mathrm{M}$ ultiple Sklerose (MS) ist die häufigste neurologische Erkrankung des jüngeren Erwachsenenalters. $\mathrm{Zu}$ den am meisten belastenden Symptomen vor allem fortgeschrittener Stadien der MS gehört die Spastik; seltener, jedoch ebenso belastend, können Schmerzphänomene auftreten. Neben der Behandlung des akuten Schubes mit Glukokortikosteroiden und der immunmodulierenden oder immunsuppressiven Langzeittherapie mit Interferon-beta oder Glatiramerazetat bzw. mit Mitoxantron hat sich die symptomatische Therapie als drittes Standbein der pharmakologischen MS-Therapie etabliert. Die deutsch-österreichisch-schweizerische Multiple Sklerose Therapie Konsensus Gruppe (MSTKG) hat generelle Empfehlungen über die Therapie der verschiedenen Symptome der MS ausgearbeitet, deren Publikation im Sommer 2004 erwartet wird (siehe auch Beitrag von Henze).

\section{Cannabinoide - \\ eine Option bei MS?}

Seit vielen Jahren liegen anekdotische Patientenberichte vor, die auf günstige Wirkungen beim Konsum von Haschisch oder Marihuana auf sowohl Spastik als auch Schmerz bei MS hinweisen. Schwierig ist die Interpretation dieser Berichte in zwei-

Basierend auf Patientenberichten und Untersuchungen mit Tiermodellen und im Reagenzglas ergaben sich eine Reihe von Hinweisen auf einen positiven Effekt von Cannabinoiden als symptomatische Therapie von Spastik und Schmerzen bei Multipler Sklerose. Mit der so genannten CAMS-Studie wurde kürzlich die erste multizentrische, randomisierte, plazebokontrollierte Phase-III-Studie über die Wirkung von Cannabinoiden bei Symptomen der MS publiziert. Im primären Studienendpunkt, der Veränderung objektiv messbarer Spastizität, die in der so genannten Ashworth-Skala ausgewertet wurde, konnte zwar kein signifikanter Behandlungseffekt der Cannabinoide festgestellt werden, bei den sekundären Endpunkten, den subjektiven Patientenberichten, zeigten sich dagegen signifikante Effekte auf Spastizität und Schmerz. Ein Hauptproblem dieser Untersuchung war die einfache Entblindung der Patienten in der aktiven Behandlungsgruppe. Die Ergebnisse der CAMS-Studie werden im Kontext mit früheren Studien, dem möglichen Wirkmechanismus sowie dem Nebenwirkungsprofil diskutiert.

erlei Hinsicht. Zum einen sind bei der Einnahme dieser psychotropen Substanzen objektive neurologische Verbesserungen schwer von der subjektiven Beurteilung der Patienten zu trennen, zum anderen sind im klinischen Verlauf der MS spontane Verbesserungen bekannt.

Um eine substanzielle klinische Besserung von subjektiven Wirkungen auf die affektive Befindlichkeit der Patienten abzugrenzen, sind seit mehr als zwei Jahrzehnten klinische Studien mit Cannabinoiden, den natürlichen Inhaltsstoffen der Pflanze Cannabis sativa, unternommen worden, davon kürzlich mit der so genannten CAMS-Studie die mit Abstand größte (Tab. 1). In vier kleinen randomisierten, doppelblinden, plazebokontrollierten Studien wurden die Effekte von Cannabinoiden auf
Spastik bei MS untersucht (Tab. 1) $(6,7,11,15)$. Obwohl jeweils über subjektive Verbesserungen berichtet wurde, konnten Wirkeffekte nicht objektiviert werden.

Anfang November 2003 wurden in der Zeitschrift Lancet die Ergebnisse der ersten multizentrischen, randomisierten, plazebokontrollierten Phase-III-Studie von Cannabinoiden zur Behandlung von Spastizität bei MS veröffentlicht (Tab. 1) (17): 667 Patienten mit klinisch stabiler MS und Spastizität wurden eingeschlossen, davon wurden $630 \mathrm{~Pa}-$ tienten in 33 klinischen Zentren in Großbritannien randomisiert. Sie erhielten für die Dauer von 15 Wochen entweder oralen Cannabisextrakt ( $\mathrm{n}$ $=211$ ), orales $\Delta^{9}$-Tetrahydrocannabinol ( $\Delta^{9}$-THC), das wichtigste psychoaktive Cannabinoid ( $n=206$ ), oder 
Plazebo ( $\mathrm{n}=213$ ), wobei die Tagesdosen abhängig waren vom Gewicht und von der Tolerierbarkeit seitens der Patienten. So lagen die Durchschnittsdosen bei 2,3 bis $8,0 \mathrm{mg}$ Cannabisextrakt und bei 3,2 bis 6,6 $\mathrm{mg} \Delta^{9}$-THC, wobei die Medikation in den ersten fünf Wochen eintitriert wurde. Primäres Studienziel war die Veränderung von Spastizität, die in der so genannten Ashworth-Skala gemessen wurde (2). Diese Skala umfasst fünf Punkte:

- $0=$ normaler Muskeltonus

- 1 = leichter Widerstand bei passiver Bewegung

- 2 = stärkerer Widerstand bei passiver Bewegung, jedoch keine eingeschränkte Beweglichkeit

- 3 = deutliche Tonuserhöhung mit eingeschränkter passiver Beweglichkeit

- 4 = Steifheit in Flexions- oder Extensionsstellung.

In der CAMS-Studie wurden zehn Muskelgruppen beidseits von einem hinsichtlich des therapeutischen Arms geblindeten Untersucher geprüft und nach der Ashworth-Skala bewertet, maximal waren 40 Punkte möglich. Die Ausgangswerte der drei Gruppen lagen zwischen 21,4 (Plazebo) und 22,6 Punkten ( $\left.\Delta^{9}-\mathrm{THC}\right)$, wobei in allen Gruppen naturgemäß die unteren
Extremitäten stärker betroffen waren als die oberen Extremitäten.

611 der 630 Patienten wurden bis zum primären Endpunkt verfolgt, in dem kein Behandlungseffekt dokumentiert werden konnte $(\mathrm{p}=$ 0,40 ). In allen drei Gruppen wurde eine Verbesserung in der AshworthSkala dokumentiert, die zwischen 0,92 (Plazebo) und 1,86 Punkten $\left(\Delta^{9}\right.$ THC) lag. Es gab dagegen einen signifikanten Behandlungseffekt auf Teile der sekundären Endpunkte. Zum einen wurde bei gehfähigen Patienten $(\mathrm{n}=278)$ vor und unter Behandlung die Zeit für die Gehstrecke von $10 \mathrm{~m}$ gemessen, wobei ein signifikanter Behandlungseffekt zugunsten $\Delta^{9}$-THC festgestellt wurde (die mittlere benötigte Zeit verkürzte sich im Vergleich zum Ausgangswert um $12 \%$ unter $\Delta^{9}$-THC $(p=0,015)$, um $4 \%$ unter Cannabisextrakt und um $4 \%$ unter Plazebo). Zusätzlich wurden die Patienten in Fragebögen über den subjektiven Behandlungseffekt auf vier MS-Symptome befragt, von denen bezüglich der Symptome Spastizität $(p=0,003)$ und Schmerz ( $p=0,003)$ signifikante Verbesserungen angegeben wurden. Ein Trend zur Verbesserung zeigte sich bei dem Symptom Tremor $(\mathrm{p}=$ 0,052), wohingegen kein Effekt auf das Symptom Blasenstörung angegeben wurde $(p=0,149)$.
Die Autoren folgern aus der CAMS-Studie, dass trotz noch immer fehlender Klasse-1-Evidenz eine symptomatische Verbesserung von Spastizität und Schmerz durch orale Cannabinoide vorliegen könnte.

Ein Hauptproblem der Studie war, dass ein Großteil der Patienten mit aktiver Medikation durch die Nebeneffekte von Cannabinoiden entblindet war, d.h. sowohl Patienten als auch behandelnde Ärzte konnten signifikant einen Großteil der Patienten mit aktiver Medikation zuordnen. Vor diesem Hintergrund sind die positiven Teilergebnisse der Studie zu relativieren. Auch ist ein Einfluss des psychotropen Effektes der Cannabinoide in dieser Studie nicht sicher auszuschließen.

\section{Beurteilung der klinischen Studien}

Die Evidenz für einen positiven Effekt von Cannabinoiden als symptomatische Therapie bei MS ist weiterhin sehr gering (8). Dem muss entgegen gehalten werden, dass insbesondere bei der Therapie der Spastik auch für die etablierten Wirkstoffe eine schwache Evidenzlage herrscht (14). Für Tizanidin liegen beispielsweise widersprüchliche Studien über dessen Effekt auf das Ausmaß der mittels der Ash-

Tab. 1 Klinische Studien mit Cannabinoiden bei MS-Patienten mit Spastizität

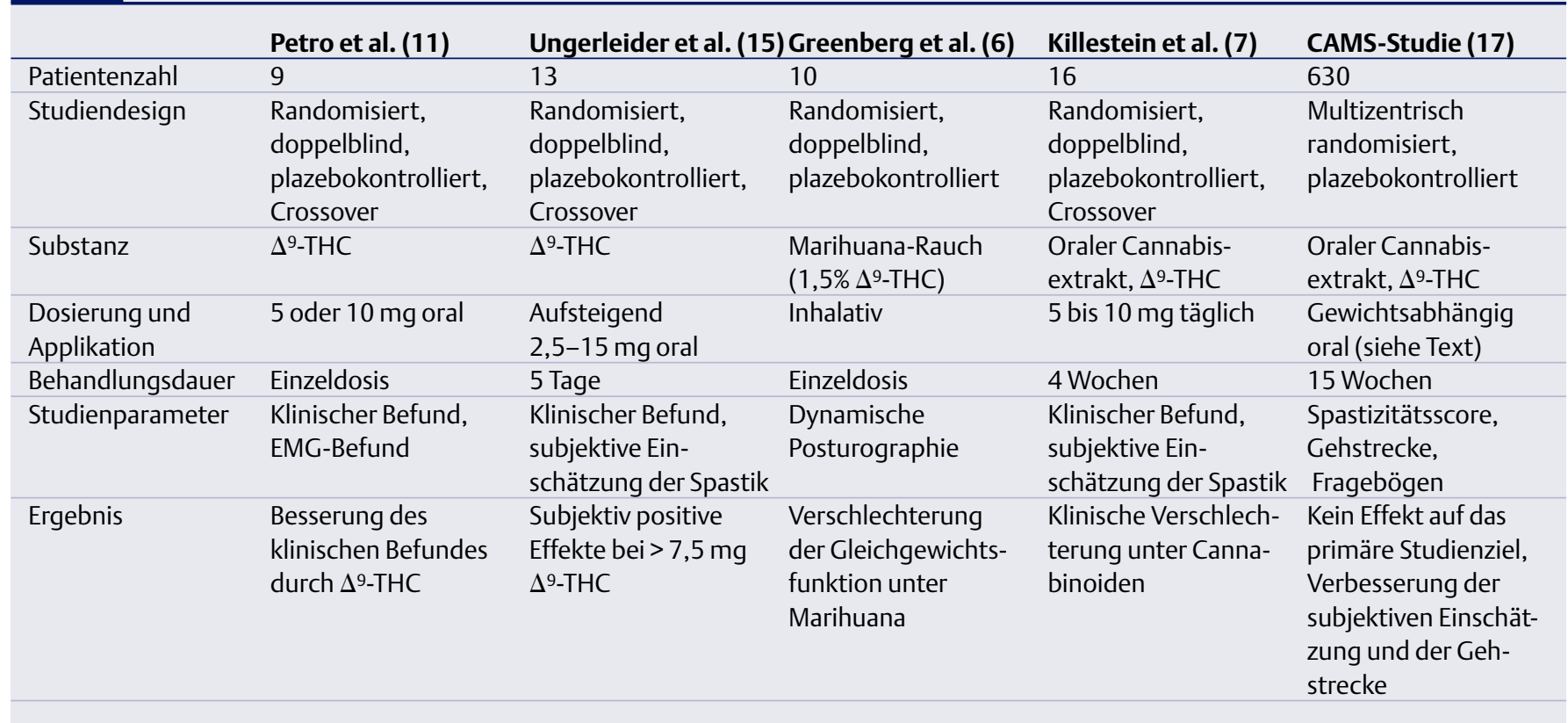


worth-Skala erfassten Spastizität vor (16). Da Spastizität pathophysiologisch ein komplexes Phänomen ist, sind deshalb möglicherweise genauere Messmethoden hilfreich, die sowohl objektive Parameter als auch subjektive Selbsteinschätzungen der Patienten beinhalten.

Ein wichtiger Aspekt bei der Beurteilung der Cannabinoide als Therapieoption ist neben deren Zusammensetzung die Dosis: während psychotrope Nebenwirkungen in der Regel bei höheren Dosen in den Vordergrund treten, könnte bei niedrigeren Dosen ein wesentlich günstigeres Nebenwirkungsprofil vorliegen. Prospektive Studien zur Klärung der Dosis mit dem optimalen Wirkungs-Nebenwirkungs-Profil sind deshalb wünschenswert.

\section{Risiken bei Cannabinoid- einnahme}

Besondere Vorsicht bei der Einnahme von Cannabinoiden erscheint bei folgenden Situationen geboten:

- wegen des Risikos der Entwicklung einer Erstmanifestation einer schizophrenen oder affektiven Psychose bei Patienten in der Adoleszenz und im jungen Erwachsenenalter

- bei familiärer Vorbelastung

- beim Auftreten affektiver (Angst, Depression) oder schizophreniformer Symptome.

Kontraindiziert ist jeglicher Konsum von Cannabinoiden bei:

- manifesten endogenen Psychosen

- manifesten Suchterkrankungen insbesondere illegaler Drogen mit der anzunehmenden NonCompliance mit Dosissteigerung und einer Neigung zur Beeinflussung des subjektiven psychischen Befindens mittels extern zugeführter Suchtmittel.

\section{Wirkmechanismen}

Die meisten Effekte der Cannabinoide werden durch Cannabinoid-Rezeptoren vermittelt, von denen bisher zwei identifiziert werden konnten: $\mathrm{CB}_{1}$ (9) und $\mathrm{CB}_{2}$ (10). $\mathrm{CB}_{1}$-Rezeptoren werden auf ZNS-Neuronen exprimiert (vornehmlich in Kleinhirn, Hippokampus und Kortex), wo sie die Freisetzung von Neurotransmittern inhibieren. Es wird angenommen, dass auf dieser Neurotransmitter-inhibierenden Eigenschaft sowohl die psychotropen Effekte der Cannabinoide als auch deren therapeutisches Potenzial bei einer Reihe neurologischer Erkrankungen wie Morbus Parkinson oder Schädel-Hirn-Trauma beruhen (4). $\mathrm{CB}_{1}$ - und $\mathrm{CB}_{2}$-Rezeptoren werden zusätzlich auf verschiedenen Immunzellen exprimiert, darunter Bund T-Lymphozyten, Makrophagen und Natürliche Killerzellen, wodurch immunologische Effekte der Cannabinoide erklärt werden könnten. Die CB-Rezeptoren wirken als so genannte G-Protein-gekoppelte Rezeptoren, d.h. die Bindung durch ihre Liganden führt zu einer Hemmung der intrazellulären Adenylatzyklase und damit zu einer verminderten Bildung von zyklischem Adenosinmonophosphat (CAMP), einem wichtigen Mediator der zellulären Regulation. Neben den pflanzlichen und synthetischen Cannabinoiden wirken auch die Endocannabinoide über die CB-Rezeptoren $(4,12)$.

Für die MS besonders interessant ist die Beobachtung, dass Cannabinoide neuroprotektive Eigenschaften zu haben scheinen (13): im Tiermodell der MS, der Experimentellen Autoimmunen Enzephalomyelitis (EAE), wurde beobachtet, dass Knockout-Mäuse, die den $\mathrm{CB}_{1}$-Rezeptor nicht besaßen, einen schwereren Verlauf der EAE mit protrahierter Behinderung aufwiesen als Kontrolltiere. Histologisch wurde ein signifikant höheres Ausmaß axonaler Schädigung in diesen $\mathrm{CB}_{1}$-Knockout-Mäusen festgestellt. Zusätzlich konnte in einem Uveitis-Modell in therapeutischer Hinsicht ein neuroprotektiver Effekt von $\mathrm{CB}_{1}$-Agonisten nachgewiesen werden (13). In einem weiteren Tiermodell, einer viral induzierten ZNS-Demyelinisierung, wurden immunregulierende Fähigkeiten von CB-Agonisten beobachtet $(1,5)$.

In den letzten Jahren wurde auch der schon seit langem von Cannabiskonsumierenden MS-Patienten berichtete positive Effekt auf die Spastik im Tiermodell bestätigt (3).

\section{Schlussfolgerung}

Zusammenfassend können Cannabinoide trotz positiver in vitro und Tiermodell-Untersuchungen und der Ergebnisse der CAMS-Studie aufgrund der schwachen Evidenzlage und wegen des Nebenwirkungsprofils gegenwärtig nicht für die Behandlung von Symptomen der MS empfohlen werden.

Auch die MSTKG empfiehlt die Gabe von Cannabinoiden zurzeit ausschließlich im Rahmen klinischer Studien. Im Fall positiver Studienergebnisse erscheint allerdings eine zeitlich begrenzte Behandlung mit Cannabinoiden unter strengen Voraussetzungen und unter Abwägung der Nebenwirkungen grundsätzlich vertretbar.

Based on patient reports, animal data and in vitro experiments, a number of hints have emerged indicating a positive effect of cannabinoids as symptomatic treatment of spasticity and pain in multiple sclerosis. The recently published CAMS study has been the first multicenter, randomised, placebo-controlled phase III trial to examine the efficacy of cannabinoids on symptoms related to MS. There was no treatment effect of cannabinoids on the primary outcome measure, a difference in the reduction of spasticity as assessed by the so-called Ashworth score. In contrast, significant effects on patientreported spasticity and pain were documented. A major problem of the study was a high degree of patient unmasking in the active treatment group. Here we discuss the results of the CAMS study in the context of previous trials, the putative mechanism of action of cannabinoids and their adverse event profile.

\section{KeyWords:}

multiple sclerosis - symptomatic

therapy - cannabinoids

\section{Literatur}

1. Arevalo-Martin A, Vela JM, Monina-Holgado E, Borrell J, Guaza C. Therapeutic action of cannabinoids in a murine model of multiple sclerosis. J Neurosci 2003; 23: 2511-2516

2. Ashworth B. Preliminary trial of carisoprodol in multiple sclerosis. Practitioner 1964; 192: 540-542

3. Baker D, Pryce G, Croxford JL, Brown P, Pertwee RG, et al. Cannabinoids control spasticity and tremor in a multiple sclerosis model. Nature 2000; 404: 84-87 
4. Croxford JL. Therapeutic potential of cannabinoids in CNS disease. CNS Drugs 2003; 17: 179-202

5. Croxford JL, Miller SD. Immunoregulation of a viral model of multiple sclerosis using the synthetic cannabinoid $\mathrm{R}(+)$ WIN55, 212. J Clin Invest 2003; 111: 1231-1240

6. Greenberg HS, Werness SA, Pugh JE, Andrus RO, Anderson DJ, et al. Short-term effects of smoking marijuana on balance in patients with multiple sclerosis and normal volunteers. Clin Pharmacol Ther 1994; 55 : 324-328

7. Killestein J, Hoogervorst EL, Reif M, Kalkers NF, Van Loenen AC, et al. Safety, tolerability, and efficacy of orally administered cannabinoids in MS. Neurology 2002; 58 : 1404-1407

8. Killestein J, Uitdehaag BM], Polman $\mathrm{CH}$. Cannabinoids in multiple sclerosis - do they have a therapeutic role? Drugs 2004; 64 : 1-11

9. Matsuda LA, Lolait SJ, Brownstin MJ, Young AC, Bonner TI. Structure of a cannabinoid receptor and functional expression of the cloned cDNA. Nature 1990; 346: 561-564

10. Munro S, Thomas KL, Abu-Shaar M. Molecular characterization of a peripheral receptor for cannabinoids. Nature 1993; 365: 61-65

11. Petro DJ, Ellenberger CJ. Treatment of human spasticity with delta 9-tetrahydrocannabinol. J Clin Pharmacol 1981; 21: 413S-416S

12. Piomelli D. The molecular logic of endocannabinoid signalling. Nature Reviews Neuroscience 2003; 4: 873-884

13. Pryce G, Ahmed Z, Hankey DJR, Jackson SJ, Croxford JL, et al. Cannabinoids inhibit neurodegeneration in models of multiple sclerosis. Brain 2003; 126: 2191-2202

14. Shakespeare DT, Boggild M, Young C, 2003. Anti-spasticity agents for multiple sclerosis (Cochrane Review). The Cochrane Library, Issue 3, v. Update Software: Oxford. 15. Ungerleider JT, Andyrsiak T, Fairbanks L, Ellison GW, Myers LW. Delta 9 THC in the treatment of spasticity associated with multiple sclerosis. Adv Alcohol Subst Abuse 1987; 7: 39-50

16. United Kingdom Tizanidine Trial Group. A double-blind, placebo-controlled trial of tizanidine in the treatment of spasticity caused by multiple sclerosis. Neurology 1994; 44: S70-S78

17. Zajicek J, Fox P, Sanders H, Wright D, Vickery J, et al. Cannabinoids for treatment of spasticity and other symptoms related to multiple sclerosis (CAMS study): multicentre randomised placebo-controlled trial. Lancet 2003; 362: 1517-1526

\section{Korrespondenzadresse:}

Dr. Oliver Neuhaus

Heinrich-Heine-Universität

Neurologie

Moorenstr. 5

40477 Düsseldorf

\section{Hitler - posttraumatische Belastungsstörung?}

Manfred Koch-Hillebrecht: Hitler. Ein Sohn des Krieges. Fronterlebnis und Weltbild. Herbig-Verlag, München, 2003. ISBN 3776623578, gebunden, 368 Seiten, $29,90 €$

Es ist eine Unsitte der Psychiater und neuerdings auch besonders der Psychologen, bekannte Krankheitsbilder wieder zu entdecken und diese Störungen oder Krankheiten auch bekannten Persönlichkeiten, möglichst post mortem, überzustülpen. Zu den Krankheitsbildern dieser Sorte gehört auch die „posttraumatische Belastungsstörung“. Überraschenderweise wird das Syndrom von dem Autor auch bei Hitler gefunden, was bei allen Störungen und Krankheiten, die Hitler tatsächlich gehabt hat, oder die ihm angedichtet werden, diese Störung am allerwenigsten zutrifft. Hitler soll durch das Trauma im ersten Weltkrieg nachhaltig gestört worden sein. Seine Entwicklung sei daraus zu erklären.

Nach der Definition (ICD 10) ist eine „posttraumatischen Belastungsstörung“ eine „verzögerte oder protrahierte Reaktion auf ein belastendes Ereignis oder eine Situation kürzerer oder längerer Dauer mit außergewöhnlicher Bedrohung oder katastrophenartigem Ausmaß, die bei fast jedem eine tiefe Verzweiflung hervorrufen würde“. Bei Hitler nun gerade ist der erste Weltkrieg zwar ein Ereignis, aber kein belastendes. Von ihm sind zahlreiche Bemerkungen zu seinem Soldatendasein authentisch überliefert, wonach der erste Weltkrieg, seine Fronterlebnisse und sein Einsatz als Meldegänger ihn tief ergriffen haben, aber voller innerer Freude und Opferbereitschaft, die von ihm nicht als belastend oder gar als Bedrohung in Erinnerung geblieben ist.

Per definitionem wird bei der posttraumatischen Belastungsstörung gefordert, dass typische Merkmale auftreten, z.B. das wiederholte Erleben des Traumas, oder ein andauerndes Gefühl von betäubt sein

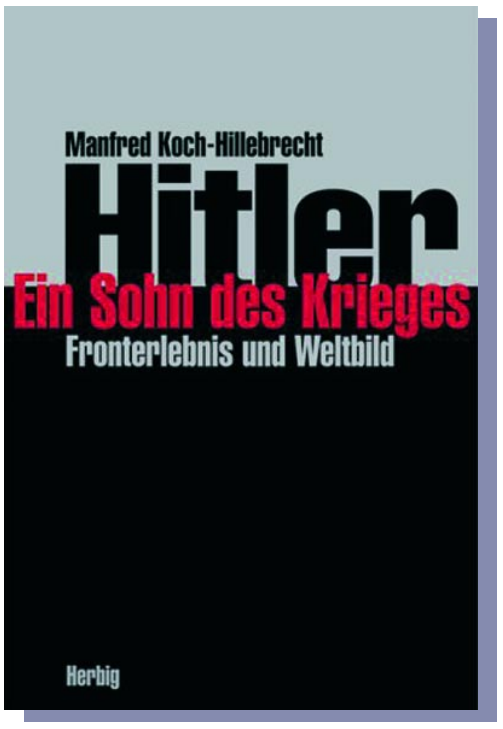

und emotionaler Stumpfheit. Ferner finden sich Gleichgültigkeit, Teilnahmslosigkeit, Freudlosigkeit, Vermeidung von Aktivitäten und Situationen, übermäßige Schreckhaftigkeit, Schlafstörungen, Angst und Depressionen. Alles das hat Hitler gerade nicht gezeigt. Seine Leistungsfähigkeit war (leider) enorm und über Jahre uneingeschränkt erhalten. Selbst kurz vor seinem Tode war Hitler noch in der Lage (trotz beginnendem Parkinson), mehrstündige Lagebesprechungen mit Frontoffizieren ad hoc abzuhalten, ohne zu ermüden und ohne Zuhilfenahme von irgendwelchen Aufzeichnungen.

Hitler war in seiner Charakterstruktur (fanatisch, paranoid, etc.) und mit seinen Begabungen (Organisationstalent, Rednergabe, hervorragendes Gedächtnis) eine hoch abnorme Persönlichkeit, die durch das Zusammenspiel dieser Eigenschaften ihn befähigte, die halbe Welt ins Unglück zu stürzen.

Prof. F. Reimer, Weinsberg 